\title{
Volatile inhibitors of atmospheric corrosion of ferrous and nonferrous metals. V. Study of the adsorption of inhibitors on steel from an aqueous electrolyte solution
}

\author{
A. I. Altsybeeva, ${ }^{1}$ V. V. Burlov, ${ }^{1}$ N. S. Fedorova ${ }^{1}$ and S. M. Reshetnikov ${ }^{2}$ \\ ${ }^{1}$ All-Russia Research Institute of Petrochemical Processes, Zheleznodorozhnyi pr. 40, \\ St. Petersburg, 192148 Russian Federation \\ ${ }^{2}$ Udmurt State University, Izhevsk, Universitetskaya 1, 426034 Russian Federation \\ E-mail:altsybeeva@yandex.ru
}

\begin{abstract}
Adsorption of ten Mannich and Schiff bases on steel from a mixed electrolyte solution $\left(30 \mathrm{mg} / \mathrm{dm}^{3} \mathrm{NaCl}+50 \mathrm{mg} / \mathrm{dm}^{3} \mathrm{Na}_{2} \mathrm{SO}_{4}\right)$ was studied. The adsorption isotherms show linear segments in the medium coverage range, which may be equally attributed to both the Frumkin and Temkin isotherms. Unfortunately, experimental data are insufficient to distinguish among them. It was demonstrated that the mechanism of action of the inhibitors in vapor phase differs from that in a model electrolyte solution and that testing of volatile inhibitors in electrolytes provides no accurate pattern of corrosion inhibition in the vapor phase. The VNKh-L-21 and VNKh-L-408 inhibitors form nanoclusters that merge on the surface into a polymolecular adsorption layer.
\end{abstract}

Key words: Schiff and Mannich bases, adsorption, mixed electrolyte, electrode surface coverage, blocking effect, Frumkin and Temkin isotherms, nanoclusters.

Received: August 10, 2013.

doi: $10.17675 / 2305-6894-2013-2-4-277-286$

\section{Experimental}

Electrochemical studies on the adsorption of inhibitors on steel were carried out in a mixed electrolyte $\left(30 \mathrm{mg} / \mathrm{dm}^{3} \mathrm{NaCl}+50 \mathrm{mg} / \mathrm{dm}^{3} \mathrm{Na}_{2} \mathrm{SO}_{4}\right)$, which is believed to simulate the electrolyte that is condensed on metal surfaces under atmospheric corrosion conditions at $100 \%$ relative humidity $[1,2]$. The adsorption was studied by measuring the differential capacitance of the double electrical layer (DEL) with an R-5021 AC bridge [3].

Measurements were carried out with parallel connection of a capacitor and a resistor. The alternating current frequency was $1 \mathrm{kHz}$. Since Faraday processes and pseudocapacitance cannot be eliminated completely, one should keep in mind that the measured capacitances give only a rough measure of the DEL capacitance and therefore the calculation will provide some affective surface coverage of an electrode with an inhibitor.

A rod shaped St3 electrode with a surface area of $1 \mathrm{~cm}^{2}$ was prepared according to a conventional procedure for electrochemical studies. It was found during the preparation and optimization of the experimental conditions that the most reproducible results were 
achieved in deaerated solutions. Therefore, introduction of an electrode and the measurements were preceded by solution deaeration with electrolytic hydrogen.

The measurements were started from some cathodic potential $(150 \mathrm{mV}$ more negative than the free corrosion potential in the blank solution). The electrode was then polarized in a stepwise fashion at a rate of $50 \mathrm{mV} / 5 \mathrm{~min}$ to a potential somewhat higher than the free corrosion potential. It was found that the dependences of capacitance on potential $(C-\varphi$ plots) are well reproducible and that capacitance is virtually potential-independent in this potential range. Therefore we calculated $\theta$ for the potential $\varphi=-0.7 \mathrm{~V}$ that is close to the free corrosion potential in this medium. The concentrations of the inhibitors were varied from $10^{-5}$ to $10^{-1} \mathrm{~mol} / \mathrm{dm}^{3}$.

The surface coverage was calculated by the formula:

$$
\theta=\left(C_{0}-C\right) /\left(C_{0}-C^{\prime}\right),
$$

where $C_{0}$ is the capacitance in the blank solution, $C^{\prime}$ is the capacitance at the maximum inhibitor concentration, and $C$ is the measured capacitance.

The potential of the working electrode (St3 steel) was measured versus a silver chloride electrode in a three-electrode cell; a platinum gauze cylinder (diameter $20 \mathrm{~mm}$, height $30 \mathrm{~mm}$ ) served as an auxiliary electrode for capacitance measurements.

The chronoamperograms ( $i-t$ curves) were recorded as described in [4]. A potential of $-0.5 \mathrm{~V}$ was applied to the electrode prior to recording the current - time decay curves. In two minutes, the current reached a constant value of $50 \mu \mathrm{A} / \mathrm{cm}^{2}$. This value remained virtually unchanged for a prolonged period of time that exceeds the time required for complete adsorption of any of the test inhibitors. The current remained virtually constant for three minutes because the electrode potential was close to the free corrosion potential in the range where the anodic process is not limited by diffusion and the electrode does not undergo passivation in the blank solution.

The measurements were started at $i_{\text {ini }}=50 \mu \mathrm{A} / \mathrm{cm}^{2}$, which corresponds to $\varphi=-0.5 \mathrm{~V}$ in the blank solution. The potential was established with a R-30 potentiostat. Note that the anodic process already occurs at this potential, although its low rate cannot yet ensure passivation. Then we determined the time required for adsorption completion. Inhibitors were added as ethanolic solutions with gradually increasing concentrations.

\section{Results and Discussion}

Data on the adsorption of the inhibitors on steel from an aqueous mixed electrolyte solution (including the measured differential DEL capacitances, calculated electrode surface coverages with the inhibitor $\theta$, and $i-t$ curves) are summarized in Tables 1 and 2 . The parameters given in the tables include:

- $\mathrm{pH}$ of a $0.3 \%$ solution of the inhibitor in aqueous ethanol $(1: 1)$;

- $W=\lg \left[\mathrm{RNH}_{3}^{+}\right] /\left[\mathrm{RNH}_{2}\right]$ (see below); 
- $\theta$ is the surface coverage of a St3 electrode at inhibitor concentration $C_{\text {inh }}=10^{-2}$ $\mathrm{mol} / \mathrm{dm}^{3}$;

- $\gamma$ is the inhibition factor for atmospheric corrosion in an environmental chamber $[5,6]$;

- $Z$ is the protective value (\%) in solution calculated by the standard formula from corrosion current data in a blank experiment and in the presence of the inhibitor $\left(C_{\text {inh }}=10^{-2} \mathrm{~mol} / \mathrm{dm}^{3}\right)$;

- $i$ is the corrosion current $(\mu \mathrm{A})$ at adsorption completion time $t$ and $C_{\mathrm{inh}}=10^{-2} \mathrm{~mol} / \mathrm{dm}^{3}$. The corrosion current in a blank experiment is $50 \mu \mathrm{A}$;

- $t$ is the adsorption completion time (s).

Table 1. Selected adsorption and physicochemical characteristics of formaldehyde derivatives.

\begin{tabular}{ccccccccc}
\hline Inhibitor & $\begin{array}{c}\text { Reaction } \\
\text { components }\end{array}$ & $\mathbf{p H}^{\boldsymbol{a}}$ & $\boldsymbol{W}^{\boldsymbol{b}}$ & $\boldsymbol{\theta}^{\boldsymbol{c}}$ & $\boldsymbol{i}^{\boldsymbol{d}}, \boldsymbol{\mu \mathbf { A }}$ & $\boldsymbol{t}^{\boldsymbol{e}}, \mathbf{s}$ & $\boldsymbol{Z}^{\boldsymbol{f}}, \boldsymbol{\%}$ & $\boldsymbol{\gamma}^{\boldsymbol{g}}$ \\
\hline VNKh-L-111 & CHA+FA & 11.65 & -2.1 & 0.70 & 8 & 50 & 84 & 220 \\
VNKh-L-112 & MP+FA & 10.15 & -3.0 & 0.68 & 9 & 90 & 82 & 250 \\
VNKh-L-113 & CHA+MP+FA & 11.51 & -2.0 & 0.52 & 20 & 80 & 60 & 335 \\
VNKh-L-405 & CHA+BTA+FA & 9.40 & -3.5 & 0.65 & 14 & 120 & 72 & 50 \\
VNKh-L-406 & MP+BTA+FA & 9.16 & -3.8 & 0.42 & 20 & 90 & 60 & 72 \\
\hline
\end{tabular}

Notes. ${ }^{a}$ For $0.3 \%$ solution in a water-ethanol mixture $(1: 1)$.

${ }^{b} \log \left[\mathrm{RNH}_{3}{ }^{+}\right] /\left[\mathrm{RNH}_{2}\right]$.

${ }^{c}$ At inhibitor concentration $C=10^{-2} \mathrm{~mol} / \mathrm{dm}^{3}$.

${ }^{d}$ At $t$ (see below) and $C=10^{-2} \mathrm{~mol} / \mathrm{dm}^{3}$.

${ }^{e}$ The time required for the adsorption to be completed.

${ }^{f}$ The protective value in solution.

${ }^{g}$ The inhibition factor in an environmental chamber.

Let us consider the possible forms of the nitrogen-containing inhibitors being studied in the electrolyte solution [7]. All the inhibitors studied are organic bases that shift the $\mathrm{pH}$ of the medium to weak or medium basic values. This implies the equilibrium:

$$
\mathrm{RNH}_{2}+\mathrm{H}_{2} \mathrm{O} \leftrightarrow \mathrm{RNH}_{3}^{+}+\mathrm{OH}^{-} .
$$

The calculated $\lg \left[\mathrm{RNH}_{3}^{+}\right] /\left[\mathrm{RNH}_{2}\right]$ values (designated as $W$ ) and the $\mathrm{pH}$ of $0.3 \%$ solutions of inhibitors in aqueous ethanol are given in Tables 1 and 2. Note that we measured the $\mathrm{pH}$ of the inhibitor solutions at $C_{\mathrm{inh}}=0.1$ and $0.5 \mathrm{wt} . \%$ and found that the $\mathrm{pH}$ varied within $\pm 0.05-0.1$. Therefore, we made the calculations for $0.3 \mathrm{wt} . \%$ concentration, which is the closest one to the inhibitor concentration $C_{\text {inh }}=10^{-2} \mathrm{~mol} / \mathrm{dm}^{3}$. The degree of protonation expressed as the concentration ratio $\left[\mathrm{RNH}_{3}{ }^{+}\right] /\left[\mathrm{RNH}_{2}\right]$ is low: there are fewer protonated species than initial molecules by two to four orders of magnitude. Thus, it is safe to say that the latter rather than protonated species are responsible for adsorption and 
corrosion inhibition in aqueous solution and that the heteroatoms serve as the adsorption sites ("anchor" atoms).

Table 2. Selected adsorption and physicochemical characteristics of benzaldehyde derivatives.

\begin{tabular}{ccccccccc}
\hline Inhibitor & $\begin{array}{c}\text { Reaction } \\
\text { components }\end{array}$ & $\mathbf{p H}^{\boldsymbol{a}}$ & $\boldsymbol{W}^{\boldsymbol{b}}$ & $\boldsymbol{\theta}^{\boldsymbol{c}}$ & $\boldsymbol{i}^{\boldsymbol{d}}, \boldsymbol{\mu \mathbf { A }}$ & $\boldsymbol{t}^{\boldsymbol{e}}, \mathbf{s}$ & $\boldsymbol{Z}^{\boldsymbol{f}}, \boldsymbol{\%}$ & $\boldsymbol{\gamma}^{\boldsymbol{g}}$ \\
\hline VNKh-L-49 & CHA+BA & 11.33 & -2.3 & 0.68 & 10 & 50 & 80 & 125 \\
VNKh-L-20 & MP+BA & 10.10 & -2.9 & 0.65 & 12 & 100 & 76 & 250 \\
VNKh-L-21 & CHA+MP+BA & 11.22 & -2.4 & 0.55 & 16 & 160 & 68 & 250 \\
VNKh-L-407 & CHA+BTA+BA & 9.05 & -3.9 & 0.40 & 16 & 95 & 68 & 18 \\
VNKh-L-408 & MP+BTA+BA & 8.70 & -5.0 & 0.52 & 18 & 180 & 64 & 125 \\
\hline
\end{tabular}

Notes. ${ }^{a}$ For $0.3 \%$ solution in a water-ethanol mixture $(1: 1)$.

${ }^{b} \log \left[\mathrm{RNH}_{3}{ }^{+}\right] /\left[\mathrm{RNH}_{2}\right]$.

${ }^{c}$ At inhibitor concentration $C=10^{-2} \mathrm{~mol} / \mathrm{dm}^{3}$.

${ }^{d}$ At $t$ (see below) and $C=10^{-2} \mathrm{~mol} / \mathrm{dm}^{3}$.

${ }^{e}$ The time required for the adsorption to be completed.

${ }^{f}$ The protective value in solution.

${ }^{g}$ The inhibition factor in an environmental chamber.

The plots of the double layer capacitance $C\left(\mu \mathrm{F} / \mathrm{cm}^{2}\right) v s$. potential $\varphi(\mathrm{V})$ of VNKH-L111 and VNKH-L-408 inhibitors are shown as examples in Figs. 1 and 2. The plots of the electrode surface coverage $\theta v s$. concentration $(\log C)$ of the same inhibitors are shown in Fig. 3.

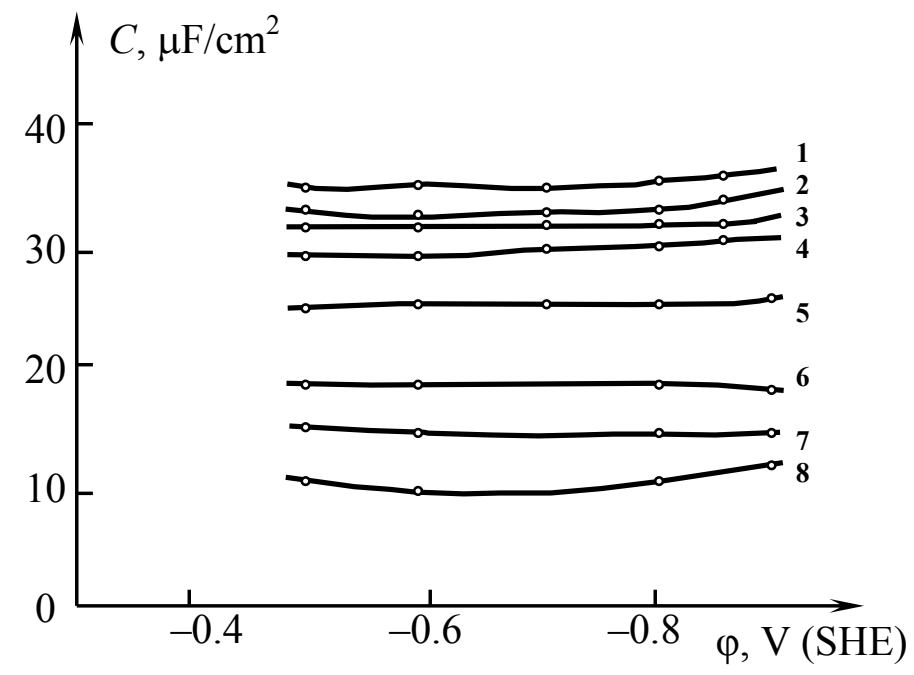

Fig. 1. Plots of double electric layer capacitance $\left(\mathrm{C}, \mu \mathrm{F} / \mathrm{cm}^{2}\right)$ of the VNKh-L-111 inhibitor on St3 steel $v s$. potential $\varphi$ for inhibitor concentrations: 1,$0 ; 2,10^{-5} ; 3,10^{-4} ; 4,10^{-3} ; 5,10^{-2} ; 6$, $2 \cdot 10^{-2} ; 7,5 \cdot 10^{-2} ; 8,10^{-1} \mathrm{~mol} / \mathrm{dm}^{3}$. 


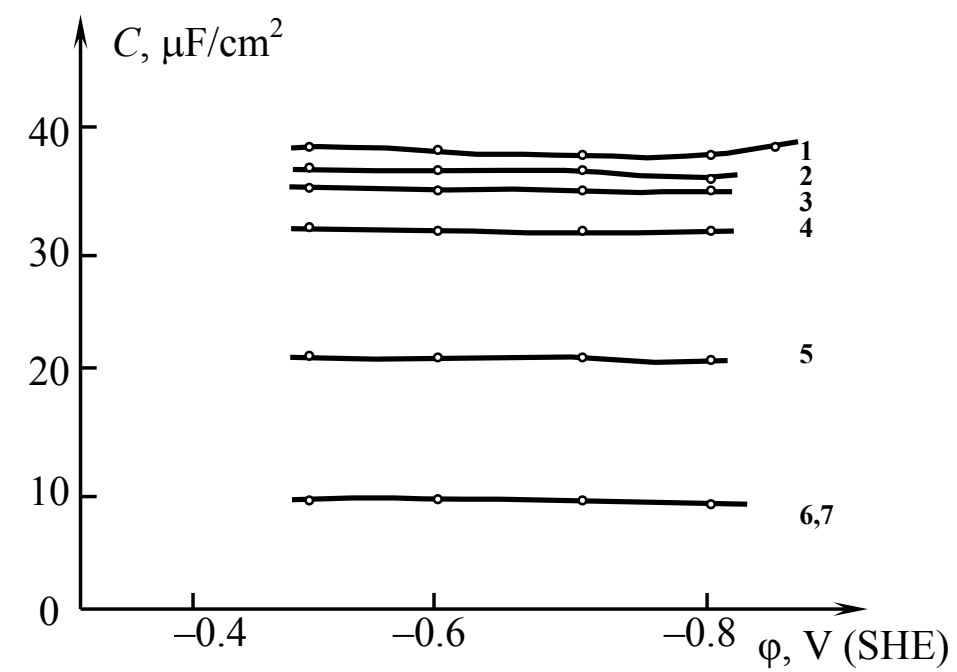

Fig. 2. Plots of double electric layer capacitance $\left(C, \mu \mathrm{F} / \mathrm{cm}^{2}\right)$ of the VNKh-L-408 inhibitor on St3 steel $v s$. potential $\varphi$ for inhibitor concentrations: 1,$0 ; 2,10^{-5} ; 3,10^{-4} ; 4,10^{-3} ; 5,10^{-2} ; 6$, $5 \cdot 10^{-2} ; 7,10^{-1} \mathrm{~mol} / \mathrm{dm}^{3}$.

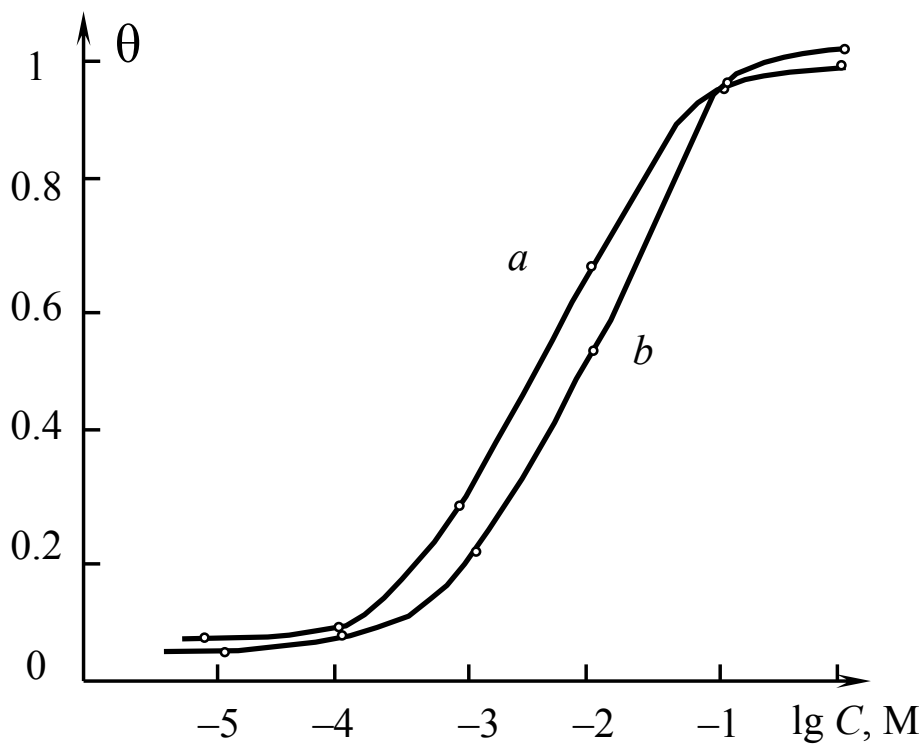

Fig. 3. Plots of surface coverage $\theta$ of a St3 electrode $v s$. concentration $(\lg C)$ of VNKh-L-111 (a) and VNKh-L-408 inhibitors $(b)$.

Preliminary experiments revealed that the concentration of an inhibitor that is poorly soluble or virtually insoluble in water can be increased by adding an inhibitor solution in ethanol to a cell with a known volume of the electrolyte. The resulting solutions do not grow turbid or opalescent, although the solubility limit of the inhibitor in water has been exceeded. The concentration of ethanol in the electrolyte thus increases to $10 \mathrm{vol} . \%$. However, alcohols are not adsorbed on iron at this concentration [8]. 
The adsorption isotherms of all the inhibitors tested show linear segments in the medium coverage range. In $\theta-\log C$ coordinates, this corresponds to the Frumkin and Temkin isotherms. An attempt to distinguish between these isotherms using the Podlovchenko-Damaskin criterion revealed that both isotherms are equally probable [9].

A specific behavior of the VNKh-L-408 inhibitor during the test should be mentioned. It follows from Fig. 2 that the capacitance initially decreases only slightly with an increase in concentration. This concentration range corresponds to a true aqueous (containing some ethanol) solution of inhibitors. Starting from $C_{\text {inh }} \sim 10^{-2} \mathrm{~mol} / \mathrm{l}$, a colloidal solution is produced (turbidity) and micelles rather than separate molecules are adsorbed from it. As a result, the capacitance decreases, while $\theta$ rapidly increases. At higher concentrations of the inhibitor, the content of micelles seems to remain constant and the coverage reaches a limit.

An additional calculation showed that the adsorption isotherms of these inhibitors can formally be described by the Frumkin isotherms with positive attractive interaction $(a \sim 1.5$ where $a$ is the attraction constant). The steep ascent of the isotherm in Fig. 3 has much to do with that for the adsorption on electrodes where the adsorption layer results from $2 \mathrm{D}$ condensation. In this case, the isotherms run virtually upright, just as we observed experimentally [3].

In the adsorption of nanoclusters (and such are colloid solutions of the VNKh-L-21 and VNKh-L-408 inhibitors), the sequence of processes includes their adsorption, coalescence in the surface layer, formation of a polymolecular adsorption layer, and saturation of the layer in such a way that a further increase in the inhibitor concentration does not increase $\theta$.

Something similar was noted in a study of the inhibitor properties of long-chain amines where reaching the critical micelle concentration, i.e., the formation of nanoclusters, sharply enhances the protective effect [10]. In acidic media, an amine is protonated and exists in solution as cations. Adsorption obeys the Frumkin isotherm with repulsive interaction of adsorbed similarly charged species in the adsorption layer. In neutral media, an amine itself is adsorbed and the Temkin isotherm is obeyed [11].

As shown in [3], a steeply ascending isotherm like that for the VNKh-L-408 inhibitor (and VNKh-L-21) is observed when repulsion in the adsorption layer - for chemisorption and physical adsorption, this is described by the Temkin and Frumkin equations, respectively - changes to attraction (i.e., positive attractive interaction). This is the case with colloid solutions of inhibitors.

The adsorptivity of surfactants can be characterized by the concentration of an adsorbate in the bulk of solution that ensures $\theta=0.5$, or by the $\theta$ value at an average "reasonable" concentration of the inhibitor $\left(\right.$ e.g., $\left.10^{-2} \mathrm{~mol} / \mathrm{dm}^{3}\right)$. We used the latter quantity to compare the inhibitors.

It can be seen in Tables 1 and 2 that, within the same series of inhibitors, the inhibitors obtained by multicomponent condensation provide lower $\theta$ values and the $i$ values on adsorption completion are higher at equal molars, which suggests their lower adsorptivity. 
It should be noted that $W$ (protonation ability) decreases in the same way. The dependences of these parameters on structure for two series of inhibitors are not so unambiguous, especially for multicomponent condensation products.

It follows from the data obtained that as the $\mathrm{pH}$ of an inhibitor solution increases (i.e., as the fraction of protonated species increases), $\theta$ at a concentration of $10^{-2} \mathrm{~mol} / \mathrm{dm}^{3}$ increases. Correlations are observed between $\theta$ at $C=10^{-2} \mathrm{~mol} / \mathrm{dm}^{3}$, corrosion current $i(\mu \mathrm{A})$ at adsorption completion, and $Z$ in an electrolyte solution, which confirms the assumption on the blocking effect of the inhibitors.

On the other hand, there are no correlations between $\theta$ in solution and $\gamma$ in the chamber as well as between $\gamma$ in the chamber and $Z$ in solution. Therefore, the mechanisms of action of the inhibitors studied differ in the vapor phase and in a model electrolyte solution and, accordingly, testing of VIACs in electrolyte solutions fails to provide an accurate idea of corrosion inhibition in the vapor phase. Apparently, an inhibitor in the vapor phase is adsorbed through its heteroatoms and blocks the metal surface, thus inhibiting atmospheric corrosion. In an electrolyte solution containing chloride and sulfate ions, the mechanism of formation of adsorption bonds is more complicated.

A positive relationship between $\theta$ in solutions and $W$ suggests the possibility of selective adsorption of protonated inhibitor species. In this case, the adsorption layer consists of non-protonated molecules adsorbed through the electrons on nitrogen (and other heteroatoms) and protonated species. The adsorption of the former will follow the Temkin isotherm, while the latter will follow the Frumkin isotherm. Perhaps, this is responsible for the impossibility of distinguishing between these isotherms on the $\theta-\log C$ curves. It is also worth noting that the blank solution contains chloride ions that are readily adsorbed on iron. Moreover, they displace sulfate and hydroxide ions, judging by their depassivating effect on iron.

The adsorption of $\mathrm{Cl}^{-}$anions (specific adsorption) charges the surface negatively (at least, the part of the surface where $\mathrm{Cl}^{-}$anions are absorbed) and favors selective adsorption of the positively charged protonated inhibitor species.

The VNKh-L-408 and VNKh-L-21 inhibitors are virtually insoluble in water; however, they form transparent solutions upon addition of their ethanolic solutions $\left(C_{\text {inh }} \leq\right.$ $\left.10^{-3} \mathrm{~mol} / \mathrm{dm}^{3}\right)$ to the blank solution. Above this concentration, a colloidal solution is formed, which is accompanied by a jump in $\theta$. At $C_{\text {inh }}>10^{-1} \mathrm{~mol} / \mathrm{dm}^{3}, \theta$ remains virtually unchanged. This isotherm is characteristic of the formation of a $2 \mathrm{D}$ compact adsorption layer [3].

Large colloid species, i.e., nanosized clusters, block the surface at concentrations $C_{\text {inh }}>$ $10^{-3} \mathrm{~mol} / \mathrm{dm}^{3}$, thus inhibiting corrosion. It follows from the $i-t$ curves (Fig. 4) that the adsorption of such large particles obeys diffusion kinetics. The formation of nanoclusters causes a stepwise increase in adsorption and the protective effect. This fact was noticed in a study of macromolecules of polymeric quaternary ammonium salts [4]. 


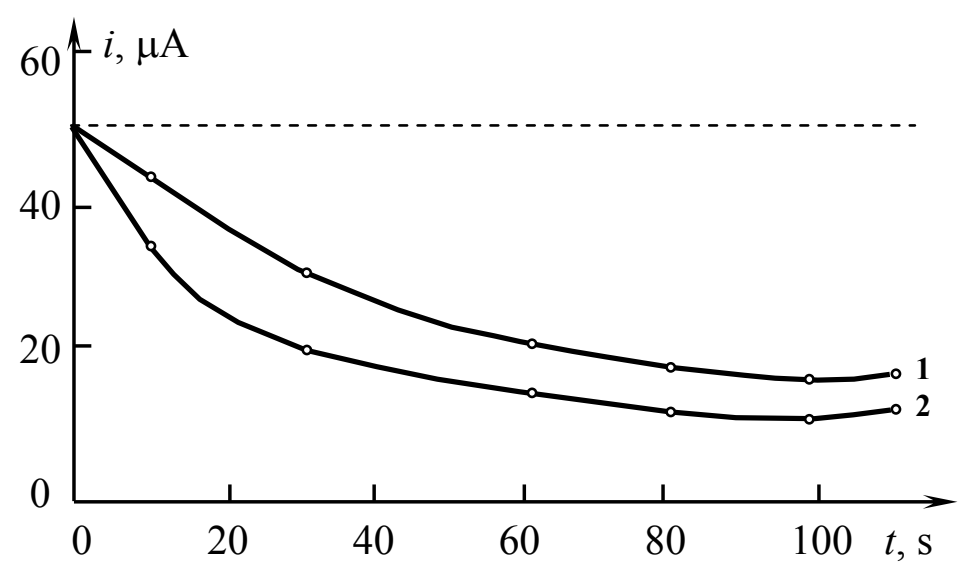

Fig. 4. Plots of corrosion current vs. adsorption time in the presence of (1) VNKh-L-408 and (2) VNKh-L-21; the data obtained without an inhibitor are indicated with a dashed line.

It is interesting that such inhibitors as VNKh-L-49 and VNKh-L-111 are adsorbed in $30-60 \mathrm{~s}$, which is characteristic of physical adsorption. It follows from the $i-t$ curves that all inhibitors obey the adsorption kinetics equation derived under the assumption that the metal surface is uniformly heterogeneous and that the inhibitors manifest a blocking effect. The VNKh-L-21 and VNKh-L-408 require the longest time for adsorption completion. This is due to diffusion limitations for large nanoclusters of these inhibitors in solution.

Thus, taking into account that the quasi-equilibrium adsorption isotherms obtained from capacitance measurements preclude us from making a definitive conclusion about the type of isotherm and hence about the adsorption mechanism and the type of species present in the adsorption layer, one can only assume that the corrosion inhibition partly results from protonated inhibitor species. Since their concentration is lower by two to four orders of magnitude than those of the initial (non-protonated) species, this suggests some selective adsorption of positively charged species above the layer of adsorbed $\mathrm{Cl}^{-}$anions.

The fact that the Frumkin isotherm is not unambiguously satisfied (except for VNKhL-21 and VNKh-L-408) provide some more evidence on the contribution of protonated species to adsorption and corrosion inhibition, which can be favored by an adsorbed layer of negatively charged chloride ions.

In addition, one should take into account that anodic dissolution most likely involves metal atoms at lattice corners, cusps, steps, and dislocations. When inhibiting the anodic dissolution of a metal, the adsorbed inhibitor affects exactly these active sites, hence general adsorption measured by $\theta$ may be lower than $Z$ (see Tables 1 and 2). 


\section{Conclusions}

1. According to the degree of protonation of the inhibitors calculated as a concentration ratio $\left[\mathrm{RNH}_{3}{ }^{+}\right] /\left[\mathrm{RNH}_{2}\right]$, the concentration of protonated species is by two to four orders of magnitude smaller than that of the original molecules. So the latter rather than protonated species are responsible for adsorption and corrosion inhibition in aqueous solution, with the heteroatoms acting as adsorption sites ("anchor" atoms).

2. The adsorption isotherms of all the inhibitors tested show linear segments in the medium coverage range. In $\theta-\log C$ coordinates, this corresponds to the Frumkin and Temkin isotherms. An attempt to distinguish between these isotherms using the PodlovchenkoDamaskin criterion revealed an equal probability of both isotherms.

3. The correlations found for the electrode surface coverage at $C=10^{-2} \mathrm{~mol} / \mathrm{dm}^{3}$, the final corrosion current $i(\mu \mathrm{A})$ (on completion of the experiment), and the protective value in an electrolyte solution confirm the assumption about the blocking effect of the inhibitors.

4. The positive relationship between the electrode surface coverage in solutions and the ratio of protonated and non-protonated forms suggests the possibility of selective adsorption of protonated inhibitor species. In this case, the adsorption layer is built of non-protonated molecules adsorbed through electrons on nitrogen (and other hetereoatoms) and protonated species. The adsorption behavior of the former will follow the Temkin isotherm, while that of the latter will follow the Frumkin isotherm. Apparently, this makes it impossible to distinguish between these isotherms in the $\theta-$ $\log C$ curves.

5. The absence of a correlation between the atmospheric corrosion inhibition factor and the protective value in an aqueous electrolyte solution suggests that the mechanisms of action of the inhibitors differ in the vapor phase and in an electrolyte model solution, hence, testing of volatile inhibitors in electrolyte solutions does not provide an accurate understanding of the inhibition mechanism in the vapor phase. Apparently, an inhibitor in the vapor phase adsorbs through its heteroatoms and blocks the metal surface, thus inhibiting atmospheric corrosion. In an electrolyte solution containing chloride and sulfate ions, the mechanism of adsorption bond formation is more complicated.

6. It was assumed that the VNKh-L-408 and VNKh-L-21 inhibitors form nanoclusters in solutions and that their adsorption behavior obeys the Frumkin isotherm with positive attractive interaction $a \sim 1.5$ (where $a$ is the attraction constant). In an electrolyte solution, the inhibitors are adsorbed and the resulting colloidal clusters coalesce to form a polymolecular adsorption layer. 


\section{References}

1. I. L. Rozenfel'd and V. P. Persiantseva, Ingibitory atmosfernoi korrozii (Inhibitors of atmospheric corrosion), Moscow, Nauka, 1985 (in Russian).

2. I. L. Rozenfel'd, Ingibitory korrozii (Corrosion inhibitors), 1977, Moscow, Khimiya (in Russian).

3. B. B. Damaskin and V. V. Batrakov, Adsorbtsiya organicheskikh soedinenii na elektrodakh (Adsorption of Organic Compounds on Electrodes), Moscow: Nauka, 1968 (in Russian).

4. S. M. Reshetnikov, Ingibitory kislotnoi korrozii metallov (Metal Corrosion Inhibitors in Acids), Khimiya, Leningrad, 1986 (in Russian).

5. A. I. Altsybeeva, V. V. Burlov, N. S. Fedorova, T. M. Kuzinova and G. F. Palatik, Int. J. Corros. Scale Inhib., 2012, no. 1, 51. doi: 10.17675/2305-6894-2012-1-1-051-064

6. A. I. Altsybeeva, V. V. Burlov, N. S. Fedorova, T. M. Kuzinova and G. F. Palatik, Int. J. Corros. Scale Inhib., 2012, no. 2, 99. doi: 10.17675/2305-6894-2012-1-2-099-106

7. N. A. Izmailov, Elektrokhimiya rastvorov (Electrochemistry of Solutions), Moscow, Khimiya, 1966 (in Russian).

8. V. V. Batrakov, G. Kh. Avad and Z. A. Iofa, Elektrokhimiya, 1968, 4, 5, 601 (in Russian).

9. B. I. Podlovchenko and B. B. Damaskin, Elektrokhimiya, 1972, 8, 3, 297 (in Russian).

10. M. A. Pletnev, A. I. Zakharov and S. M. Reshetnikov, Vestn. Udmurt. Gos. Univ. 2008, Series 4, 2, 12 (in Russian).

11. Z. A. Iofa, Elektrokhimiya, 1975, 11, 10, 1601 (in Russian). 